\title{
Contextual and sequential effects on judgments of sweetness intensity
}

\author{
HENDRIK N. J. SCHIFFERSTEIN and JAN E. R. FRIJTERS \\ Wageningen Agricultural University, The Netherlands
}

\begin{abstract}
Presenting stimuli from skewed concentration distributions affects mean responses on category scales. However, if the number of categories on the response scale is increased, the degree of separation between the mean responses obtained for a positively as opposed to a negatively skewed concentration distribution diminishes. The present study investigates the effect of skewed concentration distributions upon ratings on a line scale and compares it to the context effect found for a 7-point category scale. In addition, sequential dependencies between consecutive stimuli and responses are investigated in order to assess their relevance in taste-intensity scaling studies. The context effects are similar for the 7-point category scale and for the line scale. The analyses of sequential effects show that both preceding responses and preceding stimuli affect current responses. However, since these two factors work in opposite directions, only a small contrast effect from the previous stimulus is significant in an overall analysis. The present study shows that even though the overall sequential effects between consecutive stimuli and responses are small, the effect of experimental context may be considerable. Since subjective context is established at the beginning of a session and sequential dependencies operate throughout the whole session, it is argued that contextual and sequential effects are only indirectly related.
\end{abstract}

\section{Context Effects}

Riskey, Parducci, and Beauchamp (1979) and Riskey (1982) showed that presenting subjects with taste stimuli from a skewed (concentration) distribution affected mean responses on a 9-point category scale. When many lowconcentration stimuli were presented (positive skew), the mean intensity ratings were higher than the ratings obtained when many high-concentration stimuli were presented (negative skew). This stimulus frequency effect does not originate from sensory adaptation (Riskey, 1982), but from changes in the function relating perceived sensations to overt responses (Mellers \& Birnbaum, 1982). According to Parducci (1974), the frequency effect results from the subjects' tendency to distribute responses uniformly over the response continuum.

Parducci (1982) and Parducci and Wedell (1986) demonstrated that the differences between the ratings obtained when presenting a positively skewed stimulus distribution and those obtained with a negatively skewed distribution diminish if the number of response categories is increased. If a line scale is conceived of as a category scale with an infinite number of categories, it might seem reasonable to conjecture that the stimulus distribution effect should be smaller for line scale responses than for

The authors gratefully acknowledge the funding given to this project by the Netherlands Organization for Scientific Research (NWO). NWO PSYCHON-Grant 560-262-032, awarded to J. E. R. Frijters, was used to support H. N. J. Schifferstein during the research period. Correspondence should be addressed to Hendrik N. J. Schifferstein, Department of Food Science, Agricultural University, Bomenweg 2, 6703 HD Wageningen, The Netherlands the responses on any category scale. Indeed, Anderson $(1982$, p. 16) reported an unpublished study on the seriousness of crimes and on the likableness of vegetables (Anderson \& Leon, 1970), in which mean responses on a 200-mm graphic rating scale showed no context effects. In an unpublished experiment by Vollmecke (1987), however, line scale judgments of sucrose sweetness showed contextual effects comparable to those found by Riskey et al. (1979) for ratings on a 9-point scale.

Two theories that try to account for context effects are Helson's (1964) adaptation-level theory and Parducci's $(1965,1974)$ range-frequency theory. According to Helson, the level of behavioral adaptation $(\mathrm{AL})$ is determined by the pooled effect of focal, background, and residual stimuli. A stimulus eliciting a sensation to which an individual is behaviorally adapted will get either a neutral response or no response. Adaptation-level theory predicts that the AL will be equivalent to a low-intensity sensation if many low-concentration stimuli are presented. Since the AL is represented by the neutral category on the response scale, the mean responses for the stimuli in this set will be higher than the responses to the same stimuli in a set with many high-intensity stimuli.

The range-frequency theory regards the mean category judgment $(C)$ given to a stimulus $\left(S_{i}\right)$ as a compromise between the range value $(R)$ and the frequency value $(F)$ of that stimulus. The range value for a stimulus follows from the psychological range: this is the intensity difference between the least and the most intense stimulus. According to the range principle, each subject divides the psychological range into categories of equal size. The number of categories used equals the number of categories 
of the response scale. The frequency principle asserts that the subject uses each response category with equal frequency. The response frequencies are assumed to be independent of stimulus range and stimulus frequency. Mathematically, the range-frequency model can be written as (e.g., Parducci \& Perrett, 1971)

$$
C_{i}=w R_{i}+(1-w) F_{i}
$$

In this equation, $w$ is a constant, indicating the relative weights of the range value and the frequency value in determining the observable judgment. High values of $w$ imply small weights for $F_{i}$ and, consequently, small context effects. The value for $w$ is assumed to be constant for all stimuli investigated and can be estimated from empirical data (see Results).

\section{Sequential Effects}

Since context affects intensity ratings, it is interesting to study whether context effects can be explained on the basis of a transfer effect from one stimulus and/or its response to the response to the next stimulus. Studies of sequential effects in loudness judgments have generally shown that current responses are contrasted with previous stimuli and assimilated toward previous responses. The nature and degree of sequential dependencies in absolute identification tasks depends on the stimulus set, the task instructions, and whether feedback is provided (Holland \& Lockhead, 1968; Mori \& Ward, 1990; Ward \& Lockhead, 1971). Sequential dependencies in studies employing a category judgment task or a magnitude-estimation task in the absence of an experimenter-induced identification function typically resemble those obtained in absolute identification tasks without feedback (Jesteadt, Luce, \& Green, 1977; Ward, 1972). In these studies, both a high-intensity stimulus $(S)$ and a high response $(R)$ on trial $t-1$ yielded a response higher than average on trial $t$. This finding can be explained as an effect of the assimilation between consecutive responses. Since $R_{t-1}$ and $S_{t-1}$ are interdependent, the effect of successive contrast between stimuli is, in this case, overshadowed by the assimilation effect between the responses. The effects of $R_{t-2}$ and $S_{t-2}$ on $R_{t}$ are typically smaller than the effects of $R_{t-1}$ and $S_{t-1}$, and often exhibit contrast instead of assimilation, depending on the experimental task.

Riskey et al. (1979) noted that solutions preceded by a higher sucrose concentration were judged significantly less sweet than the same samples preceded by a lower concentration. Similarly, the judged saltiness intensity of a $\mathrm{NaCl}$ solution is increasingly overestimated after an increasing number of preceding sucrose solutions (Kroeze, 1983; Schifferstein \& Frijters, 1992). Kamenetzky (1959) found a successive contrast effect with regard to the affective judgments of foods. Bruvold (1970), however, found no contrast effect in the successive quality judgments of water samples. Apparently, the stimulus contrast effect in taste research is mostly larger than the response assimilation effect, leading to a negative association between $S_{t-1}$ and $R_{t}$.

A conceptual framework for the study of sequential effects is shown in Figure 1. At trial $t-1$, the tasting of stimulus $S_{i^{\prime}}$ elicits a momentary sensation with intensity $I_{i, t-1}$. The intensity of the sensation perceived is then subsequently transformed into a momentary response $R_{i, t-1} . I_{i, t-1}$ is subject to variation caused by noise in the perceptual system and corresponds to a value of a normal probability distribution on the sensory continuum.with mean $\bar{I}_{i}$. Similarly, $R_{i^{\prime} t-1}$ is subject to variation and corresponds to a value of a normal distribution on the response continuum with mean $\bar{R}_{i}$, When stimulus $S_{i}$ is tasted at the subsequent trial $(t)$, its internal representation $\left(I_{i, t}\right)$ and/or the response given $\left(R_{i, t}\right)$ may be affected by the previously perceived sensation or the previous response. The analysis of sequential dependencies tries to assess the relevance of the current and the previously perceived sensations and the previous responses in determining the current response, $R_{i, t}$. Since $I_{i, t}$ cannot be quantified directly, $I_{i, t}$ is assumed to depend only on $S_{i}$, and can therefore be represented by $\overline{I_{i}}$ during data analysis. However, theoretically, the sequential dependencies found may originate both from changes in the psychophysical transformation, thereby affecting the internal representation of $S_{i}$, and from changes in the response output"

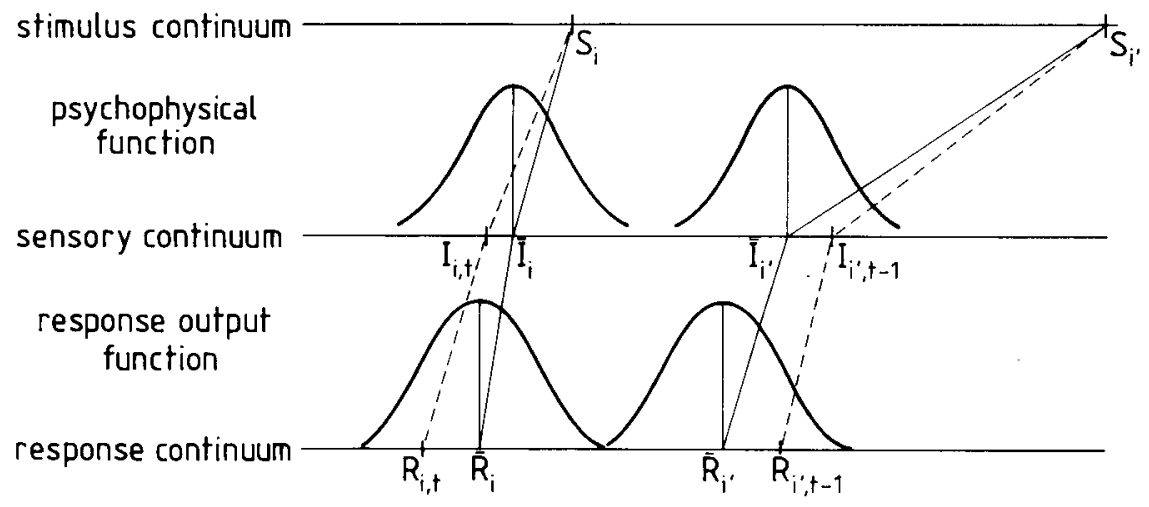

Figure 1. Interrelationships between stimuli, momentary perceived intensities, and momentary responses. Perceived intensities and responses are assumed to be symmetrically distributed on the sensory and the response continuum, respectively. 
function. The data analysis cannot discriminate between these two potential sources of sequential dependencies.

In the present study, contextual and sequential effects are studied in two experiments assessing sucrose sweetness. In the first experiment, two types of response scales are used (a line scale and a 7 -point category rating scale) in order to compare these scales with regard to their sensitivity to manipulations of experimental context. The second experiment was designed to enable a study of sequential dependencies in a line scale judgment task.

\section{METHODS}

\section{Experiment 1}

Subjects. Twenty-four untrained, unpaid volunteers, 13 men and 11 women, ranging in age from 20 to 26 years, participated in the experiment. All were students of the Agricultural University. The subjects were naive with respect both to the substances used and to the purpose of the study.

Stimuli. The stimuli were solutions of sucrose (Merck 7651) in demineralized water. The sucrose concentrations were $0.10,0.20$, $0.40,0.80$, and $1.60 \mathrm{M}$. For reasons of standardization, subjects were presented with references. The first stimulus of the reference pair was water, and the second stimulus was a solution of $1.80 \mathrm{M}$ sucrose.

Solutions were prepared at least $24 \mathrm{~h}$ before tasting and were stored in a dark, refrigerated room at $4^{\circ} \mathrm{C}$ for no longer than six days.

Design. Five experimental sucrose solutions were used to obtain two different stimulus distributions (see Table 1). In the positively skewed distribution, low concentrations were most frequent. In the negatively skewed distributions, solutions high in sucrose level were most frequent. The subjects were randomly assigned either to the positively skewed or to the negatively skewed context.

Procedure. The subjects were instructed to judge the intensity of the perceived sweetness. The instructions emphasized that only the sweetness intensity was to be judged. The hedonic value and side tastes of the stimuli were to be disregarded. In the instructions, the minimum sweetness and the maximum sweetness were defined as equal to the sweetness intensities of the two stimuli of the reference pair (i.e., water and $1.80 \mathrm{M}$ sucrose, respectively). The references were tasted at the beginning of the session and after the 25 th stimulus.

During the experimental session, the 25 stimuli from one stimulus distribution were evaluated twice $(2 \times 25=50$ stimuli $)$. Each subject used both response scales; both scales measured $150 \mathrm{~mm}$. The subjects were randomly assigned to one response-scale sequence. The left and right ends of the response scales were labeled 'not sweet at all' and 'extremely sweet.' Half the subjects within each context made their judgments on a line scale during the first part of the session, and used a 7-point category rating scale during the second part of the session (line-category sequence). The other half of the subjects first used the category rating scale and then the line scale (category-line sequence).

Table 1

Frequency of Presentation of Each of Five Sucrose Concentrations in the Two Experiments

\begin{tabular}{lrccccc}
\hline & \multicolumn{5}{c}{ Sucrose Concentration (M) } & \\
\cline { 2 - 6 } \multicolumn{1}{c}{ Context } & 0.10 & 0.20 & 0.40 & 0.80 & 1.60 & Experiment \\
\hline Positive skew & 11 & 7 & 4 & 2 & 1 & 1 \\
Uniform & 5 & 5 & 5 & 5 & 5 & 2 \\
Negative skew & 1 & 2 & 4 & 7 & 11 & 1 \\
\hline
\end{tabular}

The subjects were requested to rinse their mouths thoroughly with demineralized water after each stimulus. The stimuli were presented at room temperature $\left(\sim 20^{\circ} \mathrm{C}\right)$ in polystyrene medicine cups. Each cup contained about $10 \mathrm{ml}$ of solution. The time interval between stimuli was $50 \mathrm{sec}$; every subject participated in one 50 -min session. The order in which the 25 stimuli of each half-session were presented was randomized.

\section{Experiment 2}

The subjects, stimuli, and procedure were similar to those used in Experiment 1. Specific differences are discussed below.

Subjects. Thirty subjects, 16 men and 14 women, ranging in age from 20 to 27 years, participated in the experiment.

Procedure. Each of the five sucrose concentrations was judged 10 times by each subject, on 150 -mm line scales, five times in the first half of the session, and five times in the second half.

Data analysis. To investigate the effect of previous stimuli on current responses, the mean average deviation of the response on trial $t$ from the overall mean response, averaged over solutions, subjects, and replications, was calculated (e.g., Holland \& Lockhead, 1968). For this analysis, the deviation of the response on trial $t$ following low-concentration stimuli $(0.1$ or $0.2 \mathrm{M}$ sucrose) or highconcentration stimuli $(0.8$ or $1.6 \mathrm{M}$ sucrose) at trial $t-k$ was calculated for $k=1,2, \ldots 6$. In the response analysis, the mean deviation of the response on trial $t$ from the overall mean response was calculated for low $(<60 \mathrm{~mm})$ and high previous responses (>90 mm).

In a simultaneous analysis (Petzold, 1981), mean responses at trial $t$ were calculated for each time lag $(k)$ as a function of the stimulus presented at trial $t-k\left(S_{t-k}\right)$ and as a function of the response given at trial $t-k\left(R_{t-k}\right)$. To reduce the number of possible responses, the responses were divided into five response categories. Each category represented one-fifth of the response scale. Responses on the left part of the scale $(<31 \mathrm{~mm})$ received category number 1 , responses on the second part of the scale $(>30 \mathrm{~mm}$ and $<61 \mathrm{~mm}$ ) were classified in category 2 , and so forth.

In a regression analysis, equations of the form:

$$
\begin{aligned}
R_{i, t}= & \beta_{t} I_{i, t}+\gamma_{t-1} R_{i, t-1}+\beta_{t-1} I_{i, t-1}+ \\
& \gamma_{t-2} R_{i^{\prime} ; t-2}+\beta_{t-2} I_{i^{\prime} ; t-2}+\ldots+e_{i, t}
\end{aligned}
$$

for the stimulus sequence . . , $S_{i^{\prime \prime}}, S_{i^{\prime}}, S_{i}$ were fitted to the data. In this equation, $R_{i, t}$ denotes the response to a stimulus $S_{i}$ at trial $t$, $I_{i, t}$ is the intensity of the sensation elicited by stimulus $S_{i}$, and $e_{i, t}$ indicates the error term. If all variables in Equation 2 are expressed on a scale with identical units, then the regression coefficients $\beta$ and $\gamma$ reflect the importance of the stimulus intensities and previous responses in determining the current response. The intensity of stimulus $S_{i}$ at trial $t\left(=I_{i, t}\right)$ can be expressed in the same units as the momentary responses $\left(=R_{i, t}\right)$ if the mean response, averaged over trials, for stimulus $S_{i}\left(\bar{R}_{i}\right)$ is considered to estimate $\bar{I}_{i}$, which represents $I_{i, t}$. Equation 2 then predicts the momentary value of the response at trial $t$ on the basis of the mean responses given to the stimulus presented at trial $t\left(\bar{R}_{i}=\bar{I}_{i}=I_{i, t}\right)$, the momentary values of previous responses $\left(R_{i, t-k}\right)$, and the mean responses given to the stimuli presented at previous trials $\left(\bar{R}_{i^{\prime}}=\bar{I}_{i^{\prime}}=I_{i, t-k}\right)$. For the special case that $\beta_{t}=1$ and $\beta_{t-k}=-\gamma_{t-k}$, Equation 2 reduces to

$$
\begin{aligned}
\boldsymbol{R}_{i, t}-\overline{\boldsymbol{R}}_{i}= & \gamma_{t-1}\left(\boldsymbol{R}_{i, t-1}-\overline{\boldsymbol{R}}_{i^{\prime}}\right) \\
& +\gamma_{t-2}\left(\boldsymbol{R}_{i^{\prime}, t-2}-\overline{\boldsymbol{R}}_{i^{\prime \prime}}\right)+\ldots+e_{i, t} .
\end{aligned}
$$

Equation 3 shows that, for this special case, Equation 2 tries to explain the deviation of the momentary value of $R_{i, t}$ from the mean response to stimulus $S_{i}\left(=\bar{R}_{i}\right)$ on the basis of the deviations of the 
preceding momentary responses to the corresponding mean responses.

In a hierarchical linear regression analysis procedure (e.g., Jesteadt et al., 1977; Ward, 1979) the dependent variable $R_{i, t}$ is predicted on the basis of an increasing number of independent variables. In the first regression equation, $I_{i, t}$ is the only independent variable. In the second equation, $I_{i, t}$ and $R_{i, t-1}$ are the independent variables. Following the hierarchical procedure, other variables are added in chronological order; the variables closest in time to $R_{i, t}$ are added first to the set of independent variables. In this way, 13 different regression equations were constructed and subsequently calculated. In order to test whether the introduction of a new independent variable significantly increased the fit of the regression model, $F$ ratios were calculated with 1 denominator degree of freedom (e.g., Williams, 1986, p. 588).

In a second hierarchical regression analysis, stimulus intensities were entered into the regression equations before the corresponding responses. In this way, the effect of the sequence in which the variables were entered upon the increase in $R^{2}$ could be studied.

Because of the interruption of the session by the tasting of the references, the sequential dependencies between Stimuli 26,27, and so forth, and Stimuli 25,24 , and so forth, were not analyzed. Instead, the results of the two sessions' halves were regarded as results of two separate sessions.

\section{RESULTS}

\section{Experiment 1}

In order to verify the nonexistence of order effects resulting from the response-scale sequence, four analyses of variance (ANOVAs) were carried out. These showed that there were no significant differences between the category ratings of subjects who had first made their judgments on line scales and those of subjects who had begun

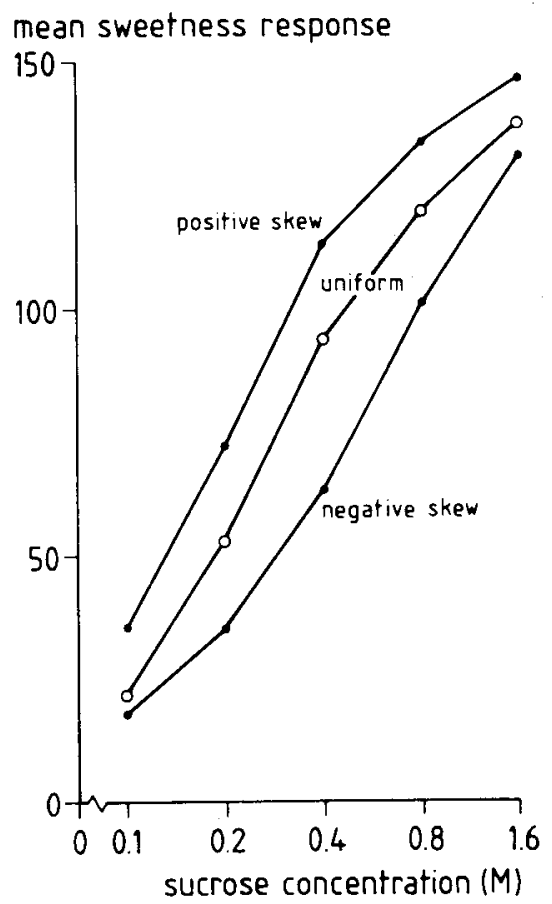

Figure 2. Mean sweetness responses on a $150-\mathrm{mm}$ line scale for five sucrose solutions, presented in three different stimulus frequency distributions.

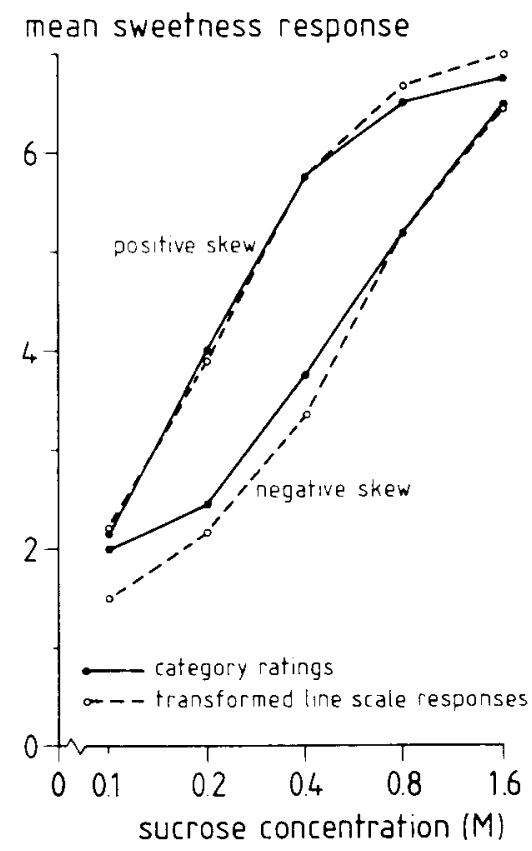

Figure 3. Mean sweetness responses on a 7-point category scale for five sucrose solutions, presented either in a positively skewed or in a negatively skewed context. The dotted lines show the mean responses obtained on a line scale after transformation to a $\chi_{\text {point }}$ scale.

a session with the category rating scale. Similarly, the responses on the line scales did not differ between response-scale sequences (line-category vs. category-line) ( $F$ test, $p>.35$ for all four conditions). Since no order effects resulted from the experimental procedure, responses were aggregated over response-scale sequences.

Figure 2 shows the mean responses on the line scale obtained in Experiment 1 (positively skewed and negatively skewed stimulus distributions) together with the mean responses obtained in Experiment 2 (uniform distribution). Obviously, context affects the sweetness judgments on the line scale. A given concentration obtains a higher sweetness response when presented within a con, text of low-concentration stimuli (positively skewed distribution) than when presented with high-concentration stimuli (negative skew). An ANOVA showed a significant effect for context in the data of Experiment 1 $[F(1,22)=25.11, p<.001]$.

The mean category ratings are depicted in Figure 3. Similar to the line scale data, mean category ratings are affected by stimulus distribution $[F(1,22)=15.51$, $p<.001]$. In order to compare the effect of context on the two response scales, the line scale data were transformed into responses on a 7-point scale by dividing the $150-\mathrm{mm}$ line into 7 equal parts. A value of 1 was assigned to responses on the first part of the scale, a value of 2 to responses on the second part of the scale, and so forth. The mean transformed responses are depicted as dashed curves in Figure 3. An ANOVA of the total data set (category rating responses and transformed line scale responses) showed no effect of response-scale type $[F(1,22)$ 
Table 2

Values of the Adaptation Level in Sucrose Concentration (M) for all Five Experimental Conditions (Helson, 1964)

\begin{tabular}{lcc}
\hline & \multicolumn{2}{c}{ Response Scale } \\
\cline { 2 - 3 } Context & Line Scale & 7-Point Scale \\
\hline Positive skew & 0.21 & 0.20 \\
Uniform & 0.29 & \\
Negative skew & 0.49 & 0.45 \\
\hline
\end{tabular}

Table 3

Estimates of the Range-Frequency Parameter $w$ (Parducci \& Wedell, 1986) for Each Concentration Level and Each Scale Type

\begin{tabular}{cccc}
\hline \multirow{2}{*}{$\begin{array}{c}\text { Sucrose } \\
\text { Concentration }\end{array}$} & \multicolumn{3}{c}{ Response Scale } \\
\cline { 2 - 3 } (M) & Untransformed & Transformed & 7-Point Scale \\
\cline { 2 - 3 } & 0.41 & 0.33 & 0.85 \\
0.1 & 0.51 & 0.52 & 0.56 \\
0.2 & 0.45 & 0.44 & 0.53 \\
0.4 & 0.57 & 0.58 & 0.77 \\
0.8 & 0.47 & 0.47 & 0.74 \\
\hline
\end{tabular}

*Estimates for $w$ were calculated for untransformed line scale responses and for responses transformed to a 7-point scale.

$=0.37, p=.55]$, nor of response scale $\times$ context interaction $[F(1,22)=0.57, p=.46]$. Therefore, the effect of the stimulus context is similar for both response scales.

Table 2 shows the values of the adaptation levels (AL) calculated for each condition (Helson, 1964). In the present instance, the AL was calculated as the concentration of sucrose giving rise to a response of 75 , the middle of the response scale (see Guilford, 1954, p. 330). The ALs were estimated using linear interpolation between the responses above and below 75 for each condition. As expected, the AL increases when more stimuli with high sucrose concentrations are presented to the subjects.

Table 3 shows the estimates of the parameter $w$ from the range-frequency model (e.g., Parducci, 1974). Frequency values $(F)$ were estimated by calculating the mean response a sample would get if subjects were only adhering to ordinal properties and trying to distribute the responses uniformly over the respective response categories. Then, assuming that the range values $(R)$ are identical for the negatively and the positively skewed stimulus distributions, $w$ can be estimated from

$$
1-w=\left(C_{i+}-C_{i-}\right) /\left(F_{i+}-F_{i-}\right),
$$

where $C_{i+}$ and $C_{i-}$ are the mean ratings for stimulus $S_{i}$ in positively and negatively skewed distributions, and $F_{i+}$ and $F_{i-}$ are the frequency values of the same stimulus in the two distributions (Parducci \& Wedell, 1986). Table 3 shows that $w$ is about 0.50 for the line scale data. However, for the category rating data, $w$ seems to depend on concentration level. For low and high sucrose levels, $w$ takes on higher values than at intermediate sucrose levels.

\section{Experiment 2}

Figure 4a shows the deviation of the response on trial $t$ from the overall mean response as a function of the stim- ulus on trial $t-k$. This figure shows that subjects tend to give high responses to stimulus $S_{i}$ if the preceding stimulus has a low intensity. Conversely, high-intensity stimuli lower the response on the next trial (contrast). This effect decreases rapidly with lag number $(k)$ and is not significant for $k>1$ (two-tailed $t$ test, $p<.05$ ).

Similar to Figure $4 a$, Figure $4 \mathrm{~b}$ shows the mean average deviation in the response on trial $t$ as a function of the response on trial $t-k$. The only statistically significant effect on $R_{i, t}$ was found for $k=6$ : a high response at trial $t-6$ seems to increase the response at trial $t$ (twotailed $t$ test, $p<.05$ ). However, since transfer effects are expected to decrease with temporal lag, and since no deviations from the overall mean are significant for $k<6$, the statistically significant result is unlikely to represent a meaningful result.

A major disadvantage of the analyses presented in Figure 4 is the suggestion that they assess the effects of previous stimuli and previous responses separately, whereas in fact they do not. Since responses and stimuli at a particular trial are highly correlated $(r \approx 0.9$ for the present data), the stimulus analysis is contaminated with response effects, and vice versa. For example, Figure 4b shows that $R_{i, t}$ tends to be higher than expected (although not significantly) after a low $R_{i, t-1}$. This deviation may be due to a small contrast effect between consecutive responses. It may also originate from a contrast effect of the preceding stimulus (as shown in Figure 4a), which is partly compensated for by an assimilation effect between consecutive responses.

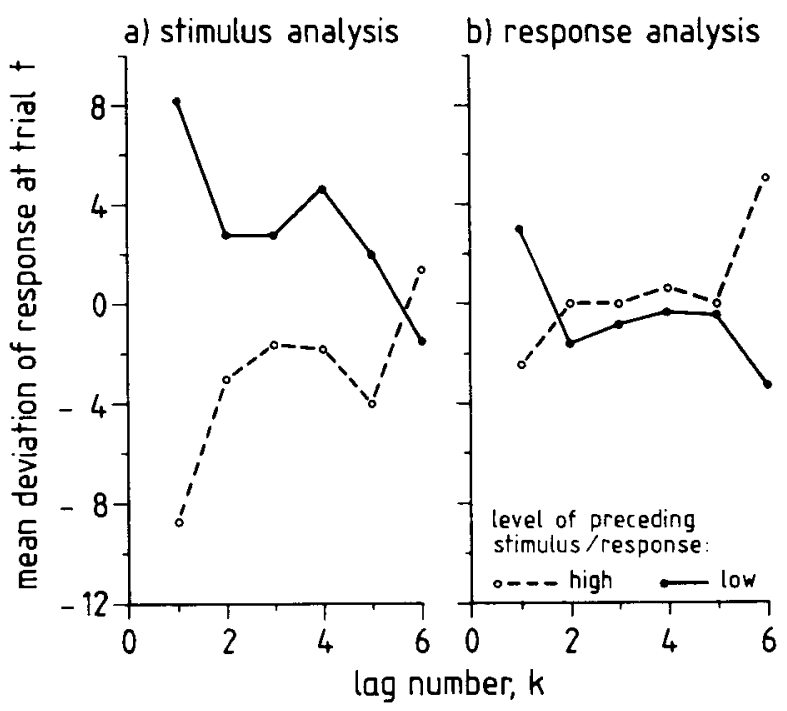

Figure 4. Sequential stimulus and response effects on sweetness judgments on a line scale. The mean deviations of the response on trial $t$ from the overall mean $\left(R_{t}-\bar{R}\right)$, averaged over stimuli, is depicted as a function of the stimulus level (panel a) and the response level (panel b) on trial $t-k$. For the stimulus analysis, the low-level preceding stimuli contained 0.1 or $0.2 \mathrm{M}$ sucrose and the high-level stimuli contained 0.8 or $1.6 \mathrm{M}$ sucrose. In the response analysis, low-level responses correspond to responses lower than 60 , whereas high-level responses are higher than 90. 


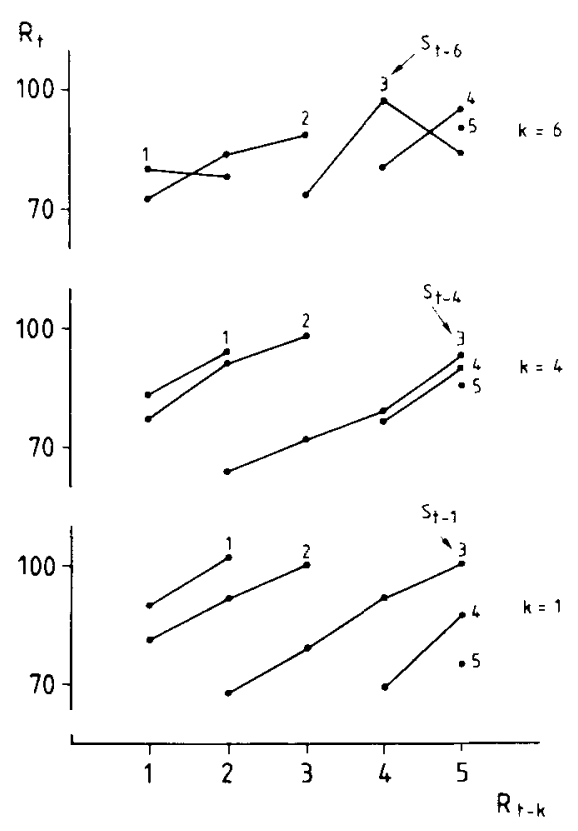

Figure 5. The mean response at trial $t\left(R_{t}\right)$ as a function of the preceding stimulus $\left(S_{t-k}\right)$ and the preceding response $\left(R_{t-k}\right)$ for $k=1$, $k=4$, and $k=6$. Responses were classified into five response categories of about equal size (see text). Mean responses at trial $t$ were used only if the number of observations for a particular combination of $S_{t-k}$ and $R_{t-k}$ exceeded 25 .

In the simultaneous analysis, effects from previous stimuli and responses are investigated simultaneously for each time lag. For each level of the previous stimulus, Figure 5 shows that the mean current response increases with increasing levels of the previous response (response assimilation). In addition, for each previous response category, the mean current responses increase with decreasing preceding stimulus levels (stimulus contrast). The size of the degree of response assimilation is reflected in the slope of the curves, whereas the stimulus contrast effect is reflected in the degree of vertical separation between the curves. As the number of lags increases, both sequential effects decrease in size, but are still clearly present for $k=4$. For $k=6$, no particularly systematic responseassimilation effect was found, which is consistent with the interpretation that the statistically significant response effect in Figure $4 b$ is due to a Type I error.

The results of the hierarchical regression analyses are shown in Table 4 . Since the absolute values of the regression coefficients decrease monotonically with the introduction of more independent variables in the regression analysis, only the coefficients obtained in the 13th regression equation are shown in the second column of Table 4 (for comparison, the regression coefficients in the third regression equation are $\beta_{t}=1.057, \gamma_{t-1}=0.358$, and $\beta_{t-1}=-0.430$ ). The regression coefficient for $I_{i, t}$ is approximately 1 . This is not surprising, since the expected value of $R_{i, t}$ is estimated by calculating $\bar{R}_{i}$, which is used as a representation of $l_{i, t}$. Regression coefficients for responses have positive signs (assimilation), whereas those for intensities have negative signs (contrast). For both types of variables, the sizes of the regression coefficients decrease with lag number. The absolute value of the regression coefficient of $I_{t-k}$ is larger than the one for $R_{t-k}$ for $k=1$, but it declines more rapidly if $k$ increases.

The consecutive increases in $R^{2}$ resulting from the introduction of a new independent variable are shown in the last two columns in Table 4 . When the variables are entered into the sequence shown in Equation 2, the values in the third column are obtained. The introduction of almost all variables significantly increases the amount of explained variance at the $p=.01$ level. The introduction of the variables $I_{t-1}$ and $I_{t-2}$ are responsible for the largest increments in $R^{2}$. However, when stimulus intensities are entered into the equation before the corresponding responses (fourth column), the responses $R_{t-1}$ and $R_{t-2}$ produce the largest increment in $R^{2}$. Now the effects of the preceding stimuli seem to be smaller than those of the preceding responses. Apparently, both the stimulus and the response to that stimulus have to be entered into the equation to obtain a large increase in $R^{2}$.

As noted by Ward $(1979$, p. 445), the problem of multicollinearity may arise in the regression analyses since $R_{i, t-k}$ and $I_{i^{\prime}, t-k}$ are not independent, but are highly correlated ( $r \approx 0.9$ for the present data). Multicollinearity may result in different increases in the amount of explained variance, depending on the sequence in which the variables are added to the regression equation. However, multicollinearity cannot account for the differences between the findings presented in the last two columns of Table 4 . Multicollinearity would predict that the first of two highly correlated variables entered into a regression equation would increase the degree of explained variance. The additional gain in explained variance by the introduction of the second variable would then be minimal, since the variance the second variable can account for is already accounted for by the first variable. Therefore, multicollinearity predicts that the preceding responses lead to the largest increases in $R^{2}$ in the third column, and that the preceding intensities lead to the largest increases in the last column. The converse, however, is shown in Table 4.

\section{Table 4}

Regression Weight Estimates and Increase in $R^{2}$ for Variables Introduced in Equation $2(n=1,140)$

\begin{tabular}{lrcc}
\hline Variable & $\begin{array}{c}\text { Regression } \\
\text { Weight }\end{array}$ & $\begin{array}{c}R^{2} \\
\text { (Sequence 1) }\end{array}$ & $\begin{array}{c}R^{2} \\
\text { (Sequence 2) }\end{array}$ \\
\hline$I_{t}$ & 1.025 & $0.9574^{*}$ & $0.9574^{*}$ \\
$R_{t-1}$ & 0.163 & 0.0002 & $0.0057^{*}$ \\
$I_{t-1}$ & -0.257 & $0.0073^{*}$ & $0.0018^{*}$ \\
$R_{t-2}$ & 0.160 & $0.0011^{*}$ & $0.0025^{*}$ \\
$I_{t-2}$ & -0.166 & $0.0017^{*}$ & $0.0003^{*}$ \\
$R_{t-3}$ & 0.110 & $0.0006^{*}$ & $0.0009^{*}$ \\
$I_{t-3}$ & -0.104 & $0.0005^{*}$ & $0.0002^{*}$ \\
$R_{t-4}$ & 0.109 & $0.0006^{*}$ & $0.0007^{*}$ \\
$I_{t-4}$ & -0.089 & $0.0003^{*}$ & $0.0002^{*}$ \\
$R_{t-5}$ & 0.078 & $0.0002^{*}$ & $0.0002^{*}$ \\
$I_{t-5}$ & -0.059 & 0.0001 & 0.0001 \\
$R_{t-6}$ & 0.078 & $0.0003^{*}$ & $0.0003^{*}$ \\
$I_{t-6}$ & -0.053 & 0.0001 & 0.0001 \\
\hline
\end{tabular}

*Increase in explained variance significant, $p<.01$. 
The finding that both $R_{t-k}$ and $I_{t-k}$ have to be entered into the regression equation to obtain the largest increase in $R^{2}$ may originate from the fact that neither the absolute levels of the preceding responses nor the absolute levels of the preceding intensities affect the current response. Instead, response is affected by the deviation of the previous responses from the mean response to that stimulus. This explanation implies that a subject giving a response lower than the mean response for that stimulus on trial $t-1$ will tend to continue to give low responses on trial $t$ (response assimilation, e.g., Ward, 1979). Equation 3 has already shown the mathematical formulation of this model. As stated before, Equation 3 implies a priori $\beta_{t}=1$ and $\beta_{t-k}=-\gamma_{t-k}$. The second column of Table 4 does, in fact, show that the regression coefficient for $I_{t}$ $\left(=\beta_{t}\right)$ is approximately equal to 1 . When the regression coefficients for previous responses are compared to those for previous stimulus intensities, they appear to be very similar. The absolute values of the two regression coefficients differ significantly only at trial $t-1$ (two-tailed $t$ test, $p<.05$ ). In this case, the difference is $0.163-0.257=-0.094$. Table 5 shows the results of a hierarchical regression analysis determining the coefficients for Equation 3. As in Table 4, the size of the regression coefficients and the increment in $R^{2}$ decreases with lag number. In addition, the coefficients are highly similar to those obtained for Equation 2.

In Table 4, deviations from the rule that $\beta_{t-k}=-\gamma_{t-k}$ demonstrate that, apart from response dependencies, stimulus dependencies are also present. The fact that stimuli exert a separate influence on consecutive responses was also noted by Jesteadt et al. (1977), who reported that the size of the regression coefficient $\left(\gamma_{t-1}\right)$ decreased with an increasing difference in stimulus intensity $\left(S_{t}-S_{t-1}\right)$. For the present study, a similar pattern of second-order dependencies was found (Figure 6). These results demonstrate that the interdependency between the response deviations on consecutive trials depends on the intensity difference between the stimuli tasted.

In summary, it can be stated that the current response largely depends on the intensity of the current stimulus (Table 4). However, effects from previous stimuli and responses on current response also occur systematically (Figure 5). Transfer effects between consecutive responses can be described by Equation 3, which shows that

Table 5

Regression Weight Estimates and Increase in $\boldsymbol{R}^{2}$ for Deviations of Momentary Responses From Overall Mean Responses Introduced in Equation $3(n=1140)$

\begin{tabular}{ccc}
\hline Time & $\begin{array}{c}\text { Regression } \\
\text { Weight }\end{array}$ & $R^{2}$ \\
\hline$t-1$ & 0.161 & $0.1334^{*}$ \\
$t-2$ & 0.161 & $0.0663^{*}$ \\
$t-3$ & 0.096 & $0.0221^{*}$ \\
$t-4$ & 0.111 & $0.0184^{*}$ \\
$t-5$ & 0.067 & $0.0061^{*}$ \\
$t-6$ & 0.083 & $0.0072^{*}$ \\
\hline
\end{tabular}

*Increase in explained variance significant, $p<.01$.

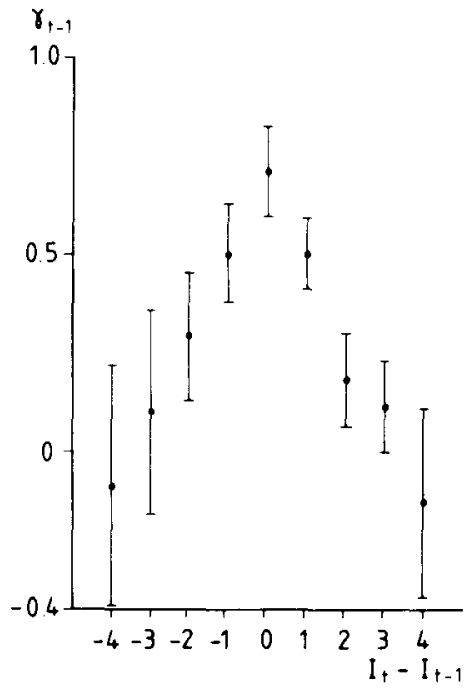

Figure 6. Estimates for $\gamma_{t-1}$ in Equation 3 as a function of the intensity difference between two consecutive stimuli. For this analysis, it was assumed that doubling the sucrose concentration always resulted in the same increase in intensity. The number on the abscissa refers to the number of times the concentration was doubled between $t-1$ and $t$. The error bars parallel to the ordinate give the $95 \%$ confidence intervals for $\gamma_{t-1}$.

the deviation of the present response from the mean response to the current stimulus depends on the size and the direction of the previous response deviations. In addition, stimuli appear to exert a separate influence on subsequent responses, as is evident from discrepancies between absolute values of $\gamma_{t-k}$ and $\beta_{t-k}$, and the secondorder dependencies.

\section{DISCUSSION}

\section{Context Effects}

The present research has shown that a line scale cannot be conceptualized as a rating scale with an infinite number of categories. If this were true, context effects should be smaller for line scale judgments than for category ratings, since context effects diminish when the number of categories is increased from 2 to 100 (Parducci, 1982; Parducci \& Wedell, 1986). In the present study, however, responses on a line scale and responses on a 7-point category scale were similarly affected by experimental context. Perhaps the line scale is used similarly to the "open category scale" (Parducci, 1982; Parducci \& Wedell, 1986). In using this scale, subjects are free to use whatever categories seem appropriate to rate the stimuli. The mean number of categories used equals five or seven, depending on the exact formulation of the task instruction (Parducci \& Wedell, 1986, p. 504).

Although the overall $F$ test did not detect a differential effect of context on the responses on the 7-point scale and for those on the line scale, Figure 3 shows that the mean ratings for 0.1 and $1.6 \mathrm{M}$ sucrose in the two contexts tend 
to be more similar for the category ratings than for the line scale judgments. This similarity of category ratings for extreme stimulus values results in a higher weight $w$ for the range value, because the context effect, that is, the stimulus frequency effect, is small (Equation 1). The estimates of $w$ in Table 3 indeed show that $w$ is higher for low and high sucrose levels for the category ratings than for the line scale judgments. These findings contradict the predictions of range-frequency theory, since this theory states that $w$ is independent of stimulus level (Equation 1). However, since $w$ is more or less constant over the concentration range for line scale judgments, it may be hypothesized that the interdependency between $w$ and sucrose level results from a response-scale-related bias such as end effects (e.g., Eriksen \& Hake, 1957; Garner, 1952, 1953; Guilford \& Dingman, 1955; Parducci, Marshall, \& Degner, 1966). If this hypothesis is correct, this finding would imply that the use of line scales is to be preferred over the use of category scales, since the former are less affected by end effects than the latter.

The present results clearly show an effect of context on line scale responses, which is in line with Vollmecke's (1987) findings. Anderson and Leon (1970), however, found no effect of stimulus distribution on line scale ratings. Possibly, the number of stimuli to be judged in their experiment (four) was insufficient to alter the reference frames the subjects used during the judgmental process. In the studies performed by Vollmecke (1987, Figure 4) and Morris and Rule (1988, Figure 2), mean ratings in different contexts approximately stabilized after 10 to 12 trials. Parducci and Wedell (1986, Figure 12) reported that the pattern of mean ratings for the first block of 10 trials was strikingly similar to the one obtained with the 50 subsequent trials. These findings suggest that if Anderson and Leon had obtained 10 or more ratings for each subject, the context effect might have been significant.

\section{Sequential Dependencies}

The sequential dependencies found in the present stimulus and response analyses (Figure 4) are different from those found in judgments of loudness (e.g., Holland \& Lockhead, 1968; Jesteadt et al., 1977; Ward \& Lockhead, 1970, 1971) and length of lines (Ward \& Lockhead, 1971). In most of these studies, current responses have been found to be assimilated toward responses and stimuli one trial back. In the present stimulus analysis, current responses were contrasted with stimuli one trial back, whereas no effect was demonstrated in the response analysis. The pattern observed in Figure 4a resembles a monotonic convergence to an asymptote, which can be accounted for by a one-stage model, assuming only effects from stimuli one trial back (Staddon, King, \& Lockhead, 1980). The deviation caused by stimuli two trials back then results from the effect of $S_{t-2}$ on $S_{t-1}$, which affects the response to $S_{t}$. However, such a simple model, incorporating only sequential effects from preceding stimuli, is not in accordance with the findings of the simultaneous analysis (Figure 5) and the regression analyses. These analyses have shown that both previous responses and previous stimuli affect current responses, but since they operate in opposite directions they partly compensate for each other (see below).

Most investigators using regression analysis to investigate sequential dependencies have used a different mathematical formulation of Equation 2. All these equations used log-transformed responses and contained an intercept. In addition, instead of using the intensity of the sensation elicited by stimulus $\left(I_{i, t-k}\right)$, the equations contained the log-transformed physical intensity of the stimulus (e.g., DeCarlo \& Cross, 1990; Green, Luce, \& Duncan, 1977; Jesteadt et al., 1977; Mori \& Ward, 1990; Ward, 1979, 1987):

$$
\begin{aligned}
\log R_{t}= & \alpha_{0}^{\prime}+\beta \beta_{t} \log S_{t} \\
& +\gamma_{t-1} \log R_{t-1}+\beta_{t-1}^{\prime} \log S_{t-1} \\
& +\gamma_{t-2} \log R_{t-2}+\beta_{t-2}^{\prime} \log S_{t-2}+\ldots+e_{t}
\end{aligned}
$$

This formulation was originally designed to account for sequential dependencies in magnitude estimation and cross-modal matching experiments, assuming a power function relating stimulus to response, and was given a theoretical basis by the response ratio hypothesis (Jesteadt et al., 1977) and Ward's (1979) fuzzy-judgment model.

Contrary to other analyses, Mori and Ward (1990) used untransformed variables in their regression analysis of absolute identification data. No explanation for this sudden change was given. In the present study, Equation 2 was used as an explanatory equation assessing the importance of each independent variable on $R_{t}$, without a theoretical basis for the explanation of the regression coefficients. The theoretical basis for the use of Equation 5 for line scale judgments or category ratings is absent, since these scales are assumed to be built up from equal intervals and not from ratios (e.g., Birnbaum, 1978).

The regression analysis of the present study showed that the separate effects of a previous stimulus intensity or its response could not account for the response deviation at" the current trial, but the combined effect of a stimulus and the corresponding response could. This finding was accounted for by Equation 3, which shows that it is not the absolute level of the current response but the deviation of that response $\left(R_{i, t}-\bar{R}_{i}\right)$ that can be explained from previous response deviations. This sequential effect, as reflected in the regression coefficients (Table 5) and the increments in $R^{2}$, decreased with lag.

This type of analysis is related to an analysis performed by Jesteadt et al. (1977). These authors calculated $\gamma_{t-1}$ in

$$
\log \left(R_{i, t} / \lambda S_{i}^{m}\right)=\gamma_{t-1} \log \left(R_{i, t-1} / \lambda S_{i^{\prime}}^{m}\right)+\alpha_{0}+e_{i, t},
$$

which is similar to:

$$
\begin{aligned}
& \log R_{i t}-\log \left(\lambda S_{i}^{m}\right) \\
& \quad=\gamma_{t-1}\left[\log R_{i^{\prime}, t-1}-\log \left(\lambda S_{i^{\prime}}^{m}\right)\right]+\alpha_{0}+e_{i, t} .
\end{aligned}
$$

In Equation $6, \lambda S_{i}^{m}$ is the prediction of $R_{i, t}$, assuming no 
response dependencies and a psychophysical power function. Equation 6 is therefore related to Equation 5 in the same way that Equation 3 (with $k=1$ ) is related to Equation 2. Morris and Rule's (1988) analysis of magnitudeestimation data is even more similar to the present approach. These authors calculated correlation coefficients between the successive deviations $\left(\log R_{i, t}-\overline{\log R_{i}}\right)$ and $\left(\log R_{i, t-1}-\overline{\log R_{i^{\prime}}}\right)$. Jesteadt et al. (1977) and Morris and Rule (1988) reported a positive association between successive response deviations. These findings are in agreement with the outcomes of the present study (see Table 5).

The experimental design that was used in the present investigation can be classified as a high-information condition (Ward, 1979) with fixed references (DeCarlo \& Cross, 1990). In a high-information condition, the amount of information transmission is high, due to the high degree of discriminability between the stimuli. Assuming that the Weber fraction for the present sucrose-concentration range equals $15 \%$ (e.g., Schutz \& Pilgrim, 1957), two successive concentration levels are separated by five just noticeable differences. This suggests that the five sucrose solutions used in the present study are easily discriminated. Increasing the discriminability between stimuli usually results in a decrease in the degree of response assimilation (Ward, 1979; Ward \& Lockhead, 1971) and, therefore, in low estimates for $\gamma_{t-1}$. In a fixed-reference condition, subjects are instructed to make their judgments relative to (a set of) fixed references. The fixed-reference instruction usually leads to lower estimates for $\gamma_{t-1}$ than an in- struction that requests subjects to make judgments relative to the previously perceived sensation and its response (DeCarlo \& Cross, 1990).

In order to relate the present analysis to the outcomes of studies employing Equation 5, $S_{t-k}$ in Equation 5 may have resulted from replacing $I_{t-k}$ with $S_{t-k}$. If the relationship between $I$ and $S$ is given by the psychophysical power function $I_{t-k}=\lambda S_{t-k}^{m}$, then Equation 5 can be written as

$$
\begin{aligned}
\log R_{t}= & \alpha_{0}+m \beta_{t} \log S_{t}+\gamma_{t-1} \log R_{t-1} \\
& +m \beta_{t-1} \log S_{t-1}+\gamma_{t-2} \log R_{t-2} \\
& +m \beta_{t-2} \log S_{t-2}+\ldots+e_{t}
\end{aligned}
$$

where $\alpha_{0}=\alpha_{0}^{\prime}+\left(\beta_{t}+\beta_{t-1}+\beta_{t-2}+\ldots\right) \log \lambda$. In this equation, $\beta_{t-k}$ and $\gamma_{t-k}$ have the same meaning as in Equation 2. If $I_{t}$ is measured in the same units as $R_{t}$, then $\beta_{t}=1$. Therefore, estimates of $\beta_{t-k}$ can be obtained by dividing each coefficient for $\log S_{t-k}$ found in any of these studies by the coefficient for $\log S_{t}(=m)$. These estimates for $\beta_{t-k}$, obtained in studies using Equation 5, can then be compared to those in Table 4 .

Table 6 shows the regression coefficients $\gamma_{t-1}$ and $\beta_{t-1}$ (corrected for the psychophysical power function exponent) for a number of studies that have employed Equation 5 . As in the present study, the regression coefficients for previous stimulus intensities have negative signs,

Table 6

A Comparison of Regression Coefiricients Obtained in Other Studies Investigating Sequential Effects

\begin{tabular}{lcclccr}
\hline \multicolumn{1}{c}{ Reference } & $k^{*}$ & Scaling $\dagger$ & Sensation & $m \neq$ & $\gamma_{t-1}$ & $\beta_{t-1}$ \\
\hline Jesteadt et al., 1977 & 1 & ME & loudness & 0.273 & 0.382 & -0.190 \\
Logue, 1976 & 1 & ME & loudness & 0.250 & 0.407 & -0.240 \\
Ward, 1979 & & & & & & \\
$\quad$ High information & 1 & ME & distance & 0.931 & 0.143 & -0.117 \\
Low information & 1 & ME & distance & 0.191 & 0.422 & 0.058 \\
High information & 1 & CMM & distance & 0.539 & 0.269 & -0.234 \\
Low information & 1 & CMM & distance & 0.381 & 0.175 & -0.205 \\
Ward, 1987I & 4 & CJ & loudness & 0.541 & 0.316 & -0.200 \\
& 4 & ME & loudness & 0.400 & 0.544 & -0.285 \\
& 4 & AME & loudness & 0.483 & 0.391 & -0.209 \\
& 4 & CMM & loudness & 0.344 & 0.332 & -0.230 \\
Mori \& Ward, 1990 & & & & & & \\
$\quad$ High information & 4 & ME & loudness & 0.30 & 0.29 & -0.13 \\
Low information & 4 & ME & loudness & 0.05 & 0.43 & -0.20 \\
High information & 4 & AI & loudness & 0.93 & 0.06 & -0.01 \\
$\quad$ Low Information & 4 & AI & loudness & 0.57 & 0.08 & 0.18 \\
DeCarlo \& Cross, 1990 & & & & & & \\
$\quad$ Fixed reference & 1 & ME & loudness & 0.572 & 0.252 & -0.114 \\
Prior reference & 1 & ME & loudness & 0.555 & 0.541 & -0.301 \\
Fixed reference & 1 & CMM & loudness & 0.696 & 0.268 & -0.172 \\
Prior reference & 1 & CMM & loudness & 0.597 & 0.417 & -0.290 \\
\hline
\end{tabular}

Note-Regression coefficients have been estimated using equations in which the variables from Equation 2 are $\log$-transformed, and in which $I$ is replaced by $S$. ${ }^{*} k=$ Number of lags used in regression analysis. $\nmid \mathrm{ME}=$ magnitude estimation, $\mathrm{CMM}=$ cross-modality matching, $\mathrm{CJ}=$ category judgment, $\mathrm{AME}=$ absolute magnitude estimation, $\mathrm{AI}=$ absolute identification. IEstimated exponent of the psychophysical power function $R_{t}=\lambda S_{t}^{m}$. §Regression coefficients corrected for the estimated exponent $m$ of the psychophysical power function. These coefficients were obtained by calculating $\beta_{t-1}^{\prime} / \beta_{t}^{\prime}$. \|Regression coefficients averaged over the outcomes of three regression analyses for three days. 
whereas those for previous responses have positive signs. In order to compare the sizes of those regression coefficients to the sizes of the regression coefficients of the present investigation, two things should be borne in mind: (1) It is assumed that the size of the regression coefficients does not depend on whether or not an intercept term is included or whether or not log-transformed or untransformed variables are fitted in the regression analysis. (2) The size of the coefficients decreases with the introduction of additional variables in the regression equation. Therefore, estimates based on equations containing only $R_{t-1}$ and $S_{t-1}$ cannot be directly compared to those estimated with more lagged variables in the regression equation.

The present values for $\gamma_{t-1}(0.358$ for $k=1 ; 0.163$ for $k=6$ ) are not particularly low or high, which may be surprising since the present experiment can be described as a high-information condition in which fixed references were used. The values for $\beta_{t-1}(-0.43$ for $k=1$; -0.257 for $k=6$ ) are more negative than most of the reported values.

DeCarlo and Cross (1990) proposed an alternative model to Equation 5 . In their model, the current response is predicted from previous stimuli in combination with an autocorrelated omitted variable (attention, memory, strategy, or motivation). The degree of autocorrelation will be large if subjects rely heavily upon immediately preceding judgments, because of accumulation of judgmental error. DeCarlo and Cross (1990) calculated the regression coefficient for $S_{t-1}$, unaffected by autocorrelation, by calculating $\beta_{t-1}^{\prime}+\gamma_{t-1} * \beta_{t}^{\prime}$. The degree of autocorrelation in their approach is given by $\gamma_{t-1}$. In a similar way, a regression coefficient for $I_{t-1}$, unaffected by autocorrelation, can be obtained by calculating $\beta_{t-1}+$ $\gamma_{t-1} * \beta_{t}$. Since $\beta_{t} \approx 1$ in the present data analysis, the coefficient for $I_{t-1}$ is estimated by $\beta_{t-1}+\gamma_{t-1}=$ $-0.257+0.163=-0.094$. In the present study, a similar calculation was performed in order to test whether previous stimuli exerted a separate effect on the current response, independent of response assimilation. The negative outcome for $\beta_{t-1}+\gamma_{t-1}$ implies a contrast effect of preceding stimuli on current responses in the present study. This finding deviates from the studies cited in Table 6, which all yielded a positive outcome (except one).

In summary, the sequential dependencies found in psychophysical taste research differ from those encountered in other sense modalities. The large contrast effect between the current response and the preceding stimuli found in the present study is in line with previous findings (e.g., Kroeze, 1983; Riskey et al., 1979) and appears to be typical for the sense of taste. In other modalities, the negative association between $R_{t}$ and $S_{t-1}$ mostly disappears after correction for response autocorrelation (DeCarlo \& Cross, 1990), whereas it persists for the sweetness judgments of the present study.

A comparison of the several types of analyses suggests that the stimulus analysis, the response analysis, and the simultaneous analysis (Figures 4 and 5) do not provide insight into the mechanism responsible for sequential ef- fects, because all these analyses use the preceding stimulus and response levels as independent variables. Regression analyses, however, have shown that the change in stimulus intensity between trial $t-1$ and trial $t$ (Figure 6) and the deviations of previous responses (Table 5) are to be preferred over the absolute stimulus and response levels in accounting for the sequential dependencies observed.

\section{The Relationship Between Sequential Dependencies and Context}

A comparison of the sequential and contextual effects encountered in the present experiments shows that, although the overall effects of a stimulus and the response to that stimulus on the next response are small (Figure 4), the effect of the total stimulus set on the mean responses is considerable (Figures 2 and 3 ). A careful examination of empirical findings and theoretical accounts demonstrates that, in several cases, outcomes of studies investigating context effects contradict the findings of those investigating sequential dependencies.

Regarding empirical findings, context effects are found to diminish if the number of categories on the response scale is increased (Parducci \& Wedell, 1986), whereas the contribution of the preceding stimulus to the predictability of the current response is found to increase (Garner, 1953).

Parducci $(1965,1974)$ accounted for context effects by stating that subjects tend to distribute their responses uniformly over the response continuum. An analysis of sequential dependencies, however, shows that consecutive responses are positively correlated. Ward and Lockhead (1971) have argued that this response assimilation may result from a guessing strategy. Since randomization of a limited set of equally spaced stimuli mostly results in small differences between consecutive stimuli, a maximization strategy with respect to the probability distribution of differences between successive stimuli would imply using only small differences between consecutive responses. According to Ward and Lockhead, subjects adopt a response strategy that is a compromise between the maximization strategy and a strategy based on the probability: distribution of successive differences. Such a strategy leads to an overestimation of the number of small stimulus differences, and the consecutive responses will therefore assimilate.

A second discrepancy between context theories and theories accounting for sequential dependencies concerns the way in which stimulus effects are accounted for. For example, Ward's (1979) fuzzy-judgment theory makes use of the (untested) assumption that the center of the internal representation of the stimulus on trial $t$ on the sensory continuum is moved away from its center on trial $t-1$. Birnbaum (1974) and Mellers and Birnbaum (1982), however, have argued that one scale of sensation underlies the psychophysical function of unidimensional, unimodal stimuli. Deviations in mean responses are attributed to task-dependent variations in the response output functions. Mellers and Birnbaum (1982) arrived at this statement because the mean responses obtained using a 
one-stimulus procedure were affected by context, whereas the scale values obtained using a two-stimulus "difference" or "ratio" judgment instruction were insensitive to contextual manipulations.

Several studies have questioned whether one single sensation scale underlies psychophysical judgment. In studies using the method of magnitude matching (Stevens \& Marks, 1980), two stimulus sets are judged on one response continuum, usually a magnitude-estimation scale. If the two stimulus sets stimulate different modalities, responses are found to vary with manipulations of the range of the stimuli stimulating own modality (Marks et al., 1988; Marks, Szczesiul, \& Ohlott, 1986). Therefore, stimulus matches (i.e., stimuli obtaining equal responses) vary with contextual manipulations. Similar shifts in responses and matches have been found in unimodal experiments where subjects judged the loudness of two sets of tones that differed in pitch (Marks, 1988) or the taste intensity of two sets of solutions eliciting different taste qualities (Rankin \& Marks, 1991). The results obtained by Marks and Warner (1991) suggest that the degree of qualitative similarity between the two stimulus sets in one experiment determines whether manipulations in one stimulus set will affect responses to the stimuli in the other set. These authors found that the shift in matching soundpressure level of two tones increased from $0 \%$ to $50 \%$ with an increase in the frequency difference between the two sets of stimuli. There was no shift in matching intensity for sets of stimuli within one critical band.

Apart from these studies on the effect of stimulus range on magnitude matchings, studies have been performed in which the interdependency between two stimulus sets was investigated by manipulating the two stimulus frequency distributions. In these studies, qualitatively different stimuli were judged independently (Parducci, Knobel, \& Thomas, 1976: size of circles vs. size of squares; Riskey \& Desor, 1980: saltiness of tomato juice vs. sweetness of cherry-flavored soft drinks), implying shifts in matching values. However, sweetness-intensity judgments for papaya and apricot beverages were highly interdependent, and were apparently similar (Vollmecke, 1987).

All these studies investigating the effect of contextual manipulations (stimulus range or frequency) on direct estimates of perceived intensity cannot demonstrate indisputably whether or not the internal representations of stimuli are affected by experimental context. Since they all use a one-stimulus procedure, changes in responses also could have resulted from changes in the response output function (e.g., Birnbaum, 1978). From an experiment employing a two-stimulus judgment task, Mellers and Birnbaum (1982, Experiment 3) concluded that scale values in a cross-modal task vary as a function of contextual manipulations. Schneider and Parker (1990) performed an experiment in which subjects judged whether the loudness difference in one stimulus pair was larger than the loudness difference in another pair of tones. The scale values calculated from the proportions obtained by this indirect scaling method showed that the stimulus matches for tones differing in frequency depend on each other's stimulus range. Algom and Marks (1990) have argued that the internal representations of stimuli may change, even if the two stimulus sets differ only with respect to the ear of presentation.

In summary, the findings obtained with direct scaling methods demonstrate that matching values obtained in experiments employing stimuli varying on more than one perceptual dimension are context-dependent. The degree of context dependency depends on the perceptual similarity between the stimulus dimensions. If the stimuli vary on one dimension or on stimulus dimensions that do not differ perceptually, matching values are not affected by contextual manipulations (Marks \& Warner, 1991). These results concur with the conclusion drawn by Mellers and Birnbaum (1982) that the internal representations of their stimulus sets did not change under different judgmental tasks using a unidimensional stimulus set. As noted above, several authors have suggested that the internal representations of stimuli varying on two or more dimensions could be affected by contextual manipulations. In the studies on sequential effects, stimuli typically varied on only one dimension. Therefore, if a theory of sequential dependencies like Ward's (1979) fuzzy-judgment model states that internal representations change as a function of previous representations, this statement does not comply with findings from experiments on context effects.

Apart from differences, some similarity exists between investigations of context effects and those of sequential effects. For example, the contrast effect found between the current response and the previous stimulus can contribute to the differences in mean ratings for positively skewed and negatively skewed concentration distributions. In the positively skewed context, many low-concentration stimuli are presented. Since studies of sequential dependencies find that low-level stimuli increase the response at the next trial, mean responses in a positively skewed context are expected to be higher than those in a uniform context.

As may be inferred from the previous discussion, sequential and contextual effects are only indirectly related. Apparently, the processes involved in the formation of the contextual reference frame are not identical to those involved in the formation of intertrial dependencies. The fact that context and sequential effects are not studied in the same experiment may have contributed to the discrepancies in empirical findings and theoretical explanations between the two research issues. Of course, the contextual reference frame within which the subjects make their judgments must be the result of previous experiences with tasting and judging preceding references and experimental stimuli. However, context is found to be established within the first 10 to 12 trials of an experiment (Morris \& Rule, 1988; Parducci \& Wedell, 1986; Vollmecke, 1987). In addition, Haubensak (1989) showed that the first 16 trials are of major importance in determining the subjective reference frame for an entire session. A shift in stimulus range after the 16th trial resulted 
in a shift in mean responses, but this effect was unable to compensate for the effect of the first 16 trials. This primacy effect was still notable after 160 additional trials.

In line with the studies discussed above, the present results suggest that the subjective context of an experiment is established in the beginning of a session and remains unchanged if the impact of the stimulus context on the subject is unaltered. In contrast, the processes forming the trial-to-trial dependencies are most prominent during the remainder of the session. Therefore, the effect of most of the trials on experimental context is expected to be minimal.

\section{REFERENCES}

Algom, D., \& Marks, L. E. (1990). Range and regression, loudness scales, and loudness processing: Toward a context-bound psychophysics. Journal of Experimental Psychology: Human Perception \& Performance, 16, 706-727.

ANDERSON, N. H. (1982). Methods of information integration theory. New York: Academic Press.

Anderson, N. H. Leon, M. (1970). Direct test for context effects in two judgmental integration tasks. Unpublished experiment, University of California, San Diego.

Birnbaum, M. H. (1974). Using contextual effects to derive psychophysical scales. Perception \& Psychophysics, 15, 89-96.

Birnbaum, M. H. (1978). Differences and ratios in psychological measurement. In N. J. Castellan, Jr., \& F. Restle (Eds.), Cognitive theory (Vol. 3, pp. 33-74). Hillsdale, NJ: Erlbaum.

BRUvoLD, W. H. (1970). Rated acceptability of mineral taste in water: III. Contrast and position effects in quality scale ratings. Journal of Experimental Psychology, 85, 258-263.

DeCarlo, L. T., CRoss, D. V. (1990). Sequential effects in magnitude scaling: Models and theory. Joumal of Experimental Psychology: General, 119, 375-396.

ERIKSEN, C. W., HAKE, H. W. (1957). Anchor effects in absolute judgments. Joumal of Experimental Psychology, 53, 132-138.

GARNER, W. R. (1952). An equal discriminability scale for loudness judgments. Journal of Experimental Psychology, 43, 232-238.

GARNER, W. R. (1953). An informational analysis of absolute judgments of loudness. Journal of Experimental Psychology, 46, 373-380.

GreEN, D M., LuCE, R. D., \& DUNCAN, J. E. (1977). Variability and sequential effects in magnitude production and estimation of auditory intensity. Perception \& Psychophysics, 22, 450-456.

Guilford, J. P. (1954). Psychometric Methods. New York: McGraw-Hill.

Guilford, J. P., Dingham, H. F. A. (1955). A modification of the method of equal-appearing intervals. American Journal of Psychology, 68, 450-454.

Haubensak, G. (1989). Primacy-Effekte in psychophysischen Urteilen. Zeitschrift fur Experimentelle und Angewandte Psychologie, 36, 42-56.

Helson, H. (1964). Adaptation-level theory. New York: Harper \& Row.

Holland, M. K., LockHEAD, G. R. (1968). Sequential effects in absolute judgments of loudness. Perception \& Psychophysics, 3 , 409-414.

Jesteadt, W., Luce, R. D., \& Green, D. M. (1977). Sequential effects in judgments of loudness. Journal of Experimental Psychology: Human Perception \& Performance, 3, 92-104

KAMENETZKY, J. (1959). Contrast and convergence effects in ratings of foods. Journal of Applied Psychology, 43, 47-52.

KroEZE, J. H. A. (1983). Successive contrast cannot explain suppression release after repetitious exposure to one of the components of a taste mixture. Chemical Senses, 8, 211-223.

LOGUE, A. W. (1976). Individual differences in magnitude estimation of loudness. Perception \& Psychophysics, 19, 279-280. (Analysis reported in Jesteadt et al., 1977.)
Marks, L. E. (1988). Magnitude estimation and sensory matching. Perception \& Psychophysics, 43, 511-525.

Marks, L. E., Stevens, J. C., Bartoshuk, L. M., Gent, J. F., RifKIN, B., \& STONE, V. K. (1988). Magnitude matching: The measurement of taste and smell. Chemical Senses, 13, 63-87.

Marks, L. E., Szczesiul, R., \& Ohlott, P. (1986). On the crossmodal perception of intensity. Journal of Experimental Psychology: Human Perception \& Performance, 12, 517-534.

Marks, L. E., \& Warner, E. (1991). Slippery context effect and critical bands. Journal of Experimental Psychology: Human Perception \& Performance, 17, 986-996.

Mellers, B. A., \& Birnbaum, M. H. (1982). Loci of contextual effects in judgment. Journal of Experimental Psychology: Human Perception \& Performance, 8, 582-601.

MorI, S., \& WARD, L. M. (1990). Unmasking the magnitude estimation response. Canadian Journal of Psychology, 44, 58-68.

MorRIS, R. B., \& RuLE, S. J. (1988). Sequential judgement effects in magnitude estimation. Canadian Journal of Psychology, 42, 69-72.

PARDuCCI, A. (1965). Category judgment: A range-frequency model. Psychological Review, 72, 407-418.

PARDUCCI, A. (1974). Contextual effects: A range-frequency analysis. In E. C. Carterette \& M. P. Friedman (Eds.), Handbook of percep tion: Vol. II. Psychophysical judgment and measurement (pp. 127. 141). New York: Academic Press.

PARDuCCI, A. (1982). Category ratings: Still more contextual effects! In B. Wegener (Ed.), Social attitudes and psychophysical measurement (pp. 89-105). Hillsdale, NJ: Erlbaum.

Parducci, A., Knobel, S., \& Thomas, C. (1976). Independent contexts for category ratings: A range-frequency analysis. Perception \& Psychophysics, 20, 360-366.

Parducci, A., Marshall, L. M., \& Degner, M. (1966). Interfertence with memory for lifted weight. Perception \& Psychophysics, 1, 83-86.

Parducci, A., \& Perrett, L. F. (1971). Category rating scales: Effects of relative spacing and frequency of stimulus values. Journal of Experimental Psychology Monographs, 89, 427-452.

PARducci, A., \& WEDELL, D. H. (1986). The category effect with rating scales: Number of categories, number of stimuli, and method of presentation. Journal of Experimental Psychology: Human Perception \& Performance, 12, 496-516.

Petzold, P. (1981). Distance effects on sequential dependencies on categorial judgments. Journal of Experimental Psychology: Human Perception \& Performance, 7, 1371-1385.

Rankin, K. M., \& Marks, L. E. (1991). Differential context effects in taste perception. Chemical Senses, 16, 617-629.

RISKEY, D. R. (1982). Effects of context and interstimulus procedures in judgments of saltiness and pleasantness. In J. T. Kuznicki, R. A Johnson, \& A. F. Rutkiewic (Eds.), Selected sensory methods: Problems and applications to measuring hedonics (pp. 71-83). Philadelphia: American Society for Testing and Materials.

RISKEY, D. R., \& DESOR, J. (1980). Independence and nonindependence between taste contexts. Unpublished manuscript, General Foods Corporation Technical Center.

Riskey, D. R., Parducci, A., \& Beauchamp, G. K. (1979). Effects of context in judgments of sweetness and pleasantness. Perception \& Psychophysics, 26, 171-176.

Schifferstein, H. N. J., \& Frijters, J. E. R. (1992). Sweetness does not habituate during a sip-and-spit experiment. Physiology \& Behavior, 51, 331-336.

SChNeIDer, B., \& PARKer, S. (1990). Does stimulus context affect loudness or only loudness judgments? Perception \& Psychophysics, 48, 409-418.

Schutz, H. G. \& Prlgrim, F. J. (1957). Differential sensitivity in gustation. Journal of Experimental Psychology, 54, 41-48.

Staddon, J. E. R., KIng, M. \& LockHEad, G. R. (1980). On sequential effects in absolute judgment experiments. Journal of Experimental Psychology: Human Perception \& Performance, 6, 290-301.

Stevens, J. C., \& Marks, L. E. (1980). Cross-modality matching functions generated by the method of magnitude estimation. Perception \& Psychophysics, 27, 379-389.

Vollmecke, T. A. (1987). The influence of context on sweetness and 
pleasantness evaluations of beverages. Unpublished doctoral dissertation, University of Pennsylvania.

WARD, L. M. (1972). Category judgments of loudnesses in the absence of an experimenter-induced identification function: Sequential effects and power-function fit. Journal of Experimental Psychology, 94, 179-184.

WARD, L. M. (1979). Stimulus information and sequential dependencies in magnitude estimation and cross-modality matching. Journal of Experimental Psychology: Human Perception \& Performance, 5 , 444-459.
WARD, L. M. (1987). Remembrance of sounds past: Memory and psychophysical scaling. Journal of Experimental Psychology: Human Perception \& Performance, 13, 216-227.

WARD, L. M., \& LockheAd, G. R. (1970). Sequential effects and memory in category judgments. Joumal of Experimental Psychology, 84, 27-34.

WARD, L. M., \& LockhEAd, G. R. (1971). Response system processes in absolute judgment. Perception \& Psychophysics, 9, 73-78.

WiLliams, R. B. G. (1986). Intermediate statistics for geographers and earth scientists. Hampshire, UK: Macmillan Education Ltd.

(Manuscript received October 9, 1991;

revision accepted for publication March 3, 1992.) 